\title{
Humane neuromuskuläre Organoide - Anwendung und Perspektive
}

\author{
JORGE MIGUEL FAUSTINO MARTINS, LAN VI NGOC NGUYEN, MINA GOUTI \\ MAX DELBRÜCK CENTRUM FÜR MOLEKULARE MEDIZIN IN DER \\ HELMHOLTZ-GEMEINSCHAFT, BERLIN
}

Organoids are miniature, organ-like structures derived from stem cells. While techniques for developing organoids for different tissues have advanced in the past decade, it has remained a challenge to simultaneously grow two different tissues into a single functional organoid. We have recently developed a 3D neuromuscular organoid that allows the simultaneous generation of spinal neurons and skeletal muscles. Here we discuss their future applications in modeling neuromuscular disorders.

DOI: $10.1007 / \mathrm{s} 12268-021-1553-0$

(C) Die Autoren 2021

Für eine kontrollierte Lokomotion ist ein komplexes neuromuskuläres Netzwerk notwendig, das rhythmische Muster der neuronalen Aktivität erzeugt, welche essenziell für viele Bewegungsabläufe sind. Defekte in diesem Netzwerk sind die Ursache für unheilbare neuromuskuläre Krankheiten, die zur Paralyse und zum Tod führen. Die Untersuchung von Krankheiten, die dieses System betreffen, wird durch die limitierte Verfügbarkeit von bewährten humanen Zellkulturmodellen erschwert.

Organoide sind miniaturisierte, vereinfachte, organähnliche Strukturen, die im Labor aus Stammzellen generiert werden. Während Methoden zur Generierung von Organoiden für verschiedene Gewebe wie das Gehirn [1, 2] im letzten Jahrzehnt weiterentwickelt wurden, ist es nach wie vor eine signifikante Herausforderung simultan zwei unterschiedliche Gewebstypen in einem einzigen funktionalen Organoid herzustellen. Wir haben vor Kurzem ein neuartiges 3D-Modell etabliert um komplexe neuromuskuläre Organoide (NMOs) herzustellen. Dieses erlaubt die simultane Generierung von Rückenmarksneuronen, Skelettmuskelzellen und Schwann-Zellen aus neuromesodermal progenitors (NMPs), die aus humanen pluripotenten Stammzellen (PS-Zellen) gewonnen wurden [3]. NMOs organisieren sich selbst in räumlich getrennte Rückenmarks- und Muskelregionen, bilden reproduzierbar funktionale neuromuscular junctions (NMJs) und kontrahieren als Antwort auf zentrale Mustergenerator-ähnliche Schaltkreise. Hier zeigen wir, dass die Langzeitkultivierung von NMOs möglich ist, wobei ihre funktionalen Komponenten erhalten bleiben. Zusätzlich diskutieren wir ihre zukünftigen Anwendungsmöglichkeiten in der Modellierung von neuromuskulären Erkrankungen.

\section{Neuromesodermale Vorläuferzellen} sind die Bausteine des

Zahlreiche kürzlich veröffentlichte Studien, haben gezeigt, dass das hintere Neuralrohr derms in enger Beziehung zueinander stehen und von derselben Linie abstammen. Dabei handelt es sich um bipotente axiale Stammzellen, die auch als neuromesodermal progenitors (NMPs) bezeichnet werden [4, 5]. Die Überlegung, dass der posteriore Embryo aus den NMPs entsteht, wurde schon 1884 vom Schweizer Anatomen Albert Kölliker nach morphologischen Beobachtungen vorgeschlagen [6], was allerdings vom vorherrschenden „Aktivierungs- und Transformationsmodell“ von Pieter D. Nieuwkoop überneuromuskulären Systems wie auch unsere Forschungsergebnisse, und die Abkömmlinge des paraxialen Meso- schattet wurde [6, 7]. Jüngere Experimente zur retrospektiven Abstammungsanalyse haben gezeigt, dass eine einzelne Zelle aus der hinteren Region des sich ausdehnenden Embryos Nachkommen erzeugen kann, die im Rückenmark sowie in der Muskulatur lokalisiert sind [4]. Somit existiert eine gemeinsame, bipotente Vorläuferzelle für Neuronen und Muskelzellen, die wichtig für die axiale Ausdehnung des Embryos und proportionales Gewebswachstum ist. NMPs wurden in der posterioren Epiblastregion aller untersuchten Wirbeltiere - vom Fisch bis hin zum Menschen - identifiziert, was daraufhin deutet, dass sie zu einer konservierten Population gehören [8].

NMPs organisieren sich selbst in 3D, um funktionale humane NMJs zu generieren

Entsprechend der Signalwege der murinen Embryonalentwicklung haben wir erfolgreich NMP-Zellen in vitro aus murinen und humanen PS-Zellen gewonnen [9, 10]. Die in vitro-Generierung dieser Zellen eröffnet neue Möglichkeiten zur Erforschung und Behandlung neuromuskulärer Erkrankungen, da hiermit ein beispielloser Zugang zur simultanen Entwicklung von neuralen und mesodermalen Zelltypen in der Petrischale geschaffen wurde. Wir haben uns vor kurzem die NMPs zur Etablierung eines neuartigen 3D-NMO-Modells zunutze gemacht, das die simultane Generierung und Selbstorganisation von Neuronen des Rückenmarks und Skelettmuskelzellen erlaubt, die funktionale NMJs bilden [3]. In 3D plattierte NMPs bilden anfangs sphärische Aggregate, die einen morphogenetischen Wandlungsprozess unterlaufen, um ein komplexes Organoid mit abgrenzenden neuroektodermalen und mesodermalen Kompartimenten zu generieren (Abb. 1A). Diese charakteristische Morphologie wird aufrechterhalten, während das Organoid heranreift. Lichtmikroskopische Aufnahmen von Organoiden verschiedener Entwicklungsstufen erlauben die Beobachtung der transparenten neuralen Region und des kontrastreicheren, kompakten Bereichs, 


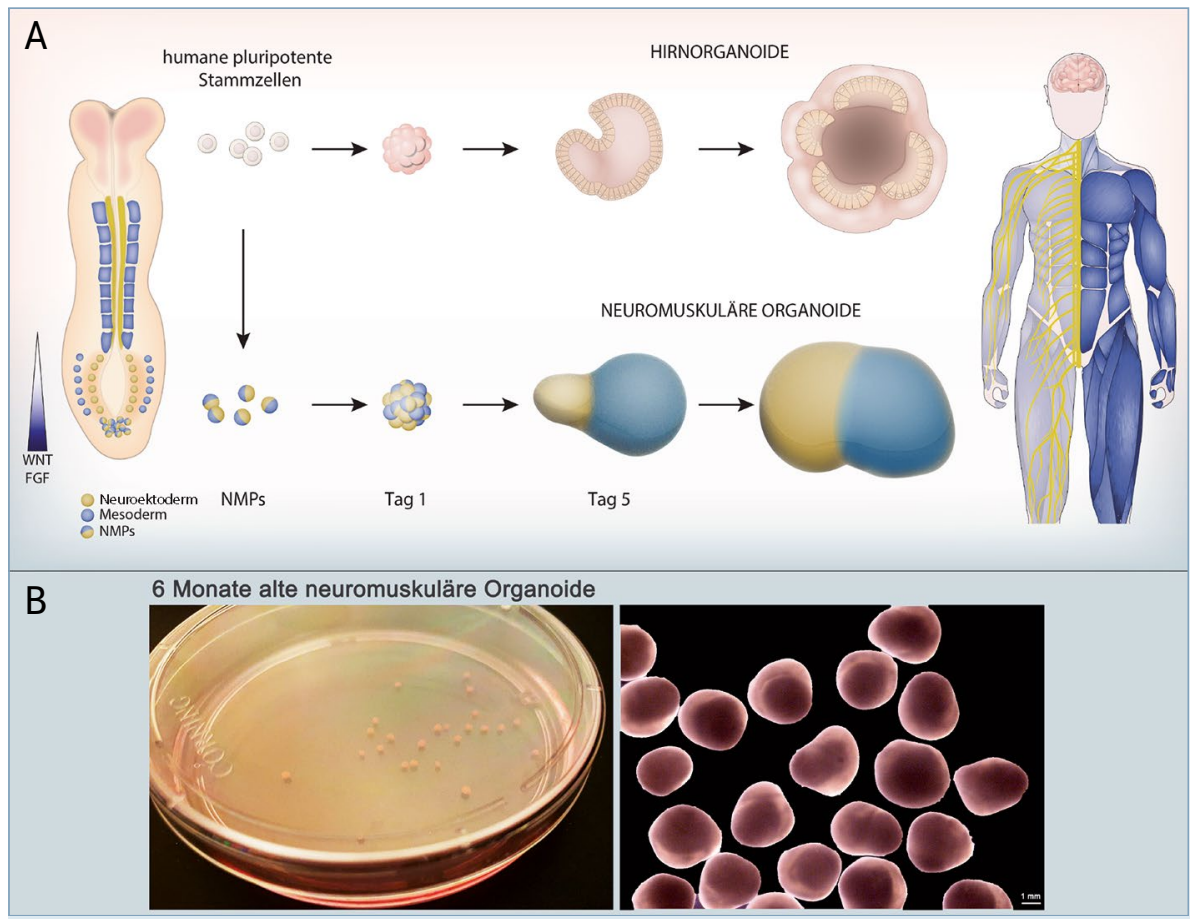

$\Delta$ Abb. 1: Erzeugung und Langzeitkultivierung von humanen Organoiden zur Modellierung der Entwicklung des zentralen Nervensystems (ZNS). A, Organoide können verwendet werden, um anteriore sowie posteriore Regionen des ZNS zu modellieren. Entsprechend der Signalwege der Embryonalentwicklung (links) können humane pluripotente Stammzellen (hPS-Zellen) in 3D kultiviert werden und organisieren sich selbst in ein Hirnorganoid mit kortikalen Faltungen. Eine Limitierung des Hirnorganoidmodells ist die Entwicklung eines nekrotischen Kerns nach längerer Kultivierung (oberer Abschnitt). Um Organoide mit posteriorer Identität herzustellen, können humane PS-Zellen zunächst WNT/FGF-Signalen ausgesetzt werden, was ihre Differenzierung in bipotente NMPs einleitet. Durch das Plattieren der NMPs unter 3D-Bedingungen differenzieren sie in selbst organisierende Vorläuferzellen des Rückenmarks und der Skelettmuskulatur, um funktionale neuromuskuläre Organoide zu generieren (unterer Abschnitt). B, NMOs können langfristig kultiviert werden. Nach sechs Monaten erreichen sie eine Größe, die mit bloßem Auge sichtbar ist (links). NMOs bewahren eine homogene Morphologie abgrenzend in neurales (heller Bereich) und Muskelgewebe (dunkler Bereich), ohne Anzeichen eines nekrotischen Kerns (rechts).

bei dem es sich um den Muskel handelt. Die NMOs können langfristig kultiviert werden, während die Gewebsregionen wachsen, interagieren und heranreifen, ohne Anzeichen von Gewebszerfall zu zeigen (Abb. 1B). Die Analyse von 180 Tage alten NMOs zeigt, dass Skelettmuskelfasern sich in einer organisierten Region des Organoids angesiedelt haben und TITIN exprimieren, während der neurale Bereich mit TUBB3 ${ }^{+}$-Neuronen angereichert ist, dessen terminale Axone das Muskelkompartiment innervieren (Abb. 2B). Die Muskelfasern besitzen eine hoch organisierte Ausrichtung und weisen eine für ausgerichtete Sarkomere typische Streifenbildung auf (Abb. 2A). Darüber hinaus wurde eine hohe Anzahl von Acetylcholinrezeptor (AChR)-Clustern durch Färbung mit $\alpha$-Bungarotoxin ( $\alpha$ BTX) detektiert, an denen sich TUBB3 ${ }^{+}$-terminale Axone zum Skelettmuskel erstrecken, womit der Erhalt der NMJs bestätigt wird (Abb. 2B und C). 180 Tage alte NMOs wiesen ein fortgeschrittenes Reifestadium auf, ersichtlich durch die weitreichende Expression des reifen Neurofilamentmarkers SMI-32 im Großteil des neuralen Bereichs. Außerdem wurde die Expression von SMI-32 ebenfalls im Muskelkompartiment detektiert, wo die Axonbahnen die Skelettmuskelfasern innervierten (Abb. 2A). Eine Population reifer Motorneuronen (MN) konnte durch die Expression von Cholinacetyltransferase (ChAT) in der spinalen Region des Organoids in der Nähe des Muskelkompartiments nachgewiesen werden (Abb. 2A). Zusammenfassend bestätigen diese Ergebnisse, dass eine Langzeitkultivierung von NMOs ohne Anzeichen von Gewebszerfall möglich ist. Die fortlaufende Interaktion der funktionalen Schlüsselkomponenten, einschließlich Skelettmuskeln und Motorneuronen, wurde durch den Nachweis funktionaler
NMJs in sechs Monate alten Organoiden bewiesen.

\section{NMOs ebnen den Weg zur personalisierten Medizin}

Neuromuskuläre Krankheiten umfassen ein breites Spektrum an Pathologien:

(i) Motorneuronerkrankungen, wie amyotrophe Lateralsklerose (ALS) und spinale Muskelatrophie (SMA),

(ii) spezifische Myopathien, wie Beckerund Duchenne- Muskeldystrophien, und

(iii) neuromuskuläre Autoimmunerkrankungen wie Myasthenia gravis [11].

Diese werden entweder durch funktionale Defekte des Nervensystems und der Skelettmuskulatur ausgelöst oder entstehen durch Defekte an den NMJs [12]. Die NMJ stellt eine hochgradig organisierte chemische Synapse dar, die zwischen Motorneuronen und Skelettmuskeln gebildet wird und mit den terminalen Schwann-Zellen einen zusätzlichen wichtigen Zelltyp enthält. In einigen der genannten Störungen ist zunächst nur die Muskulatur oder die neurale Komponente betroffen. In vielen Fällen ist es allerdings schwierig, die primäre Ursache und den Zelltypen zu identifizieren, der als erstes dysfunktional ist. Die diversen genetischen Ursachen und unterschiedlich einsetzenden Krankheitsverläufe der lähmenden neuromuskulären Erkrankungen standen bisher der Entwicklung von patientenspezifischen Therapeutika im Weg.

Die Herstellung patientenspezifischer neuromuskulärer Organoide ermöglicht uns die Untersuchung des genauen Ursprungs und des Fortschreitens der Krankheiten, die die NMJs betreffen. Der einzigartige Vorteil des Systems besteht darin, dass erstmalig der Zugang zu einem vollständig von Patienten stammenden NMJ-3D-Modell geschaffen wird. Schließlich erlauben patientenspezifische NMOs einen tieferen Einblick in die pathologischen Mechanismen von neuromuskulären Erkrankungen, indem sie den Zugang zu zuvor - aufgrund des Mangels an Modellen mit humaner NMJ-Biologie -unzugänglichen Merkmalen eröffnen.

NMOs weisen eine funktionale Aktivität auf und können, wie wir hier gezeigt haben, langfristig ohne Verlust der Schlüsselkomponenten des neuromuskulären Systems kultiviert werden. Diese Eigenschaften, kombiniert mit der Reproduzierbarkeit des in vitroProtokolls, machen NMOs sowohl zu einem attraktiven Modell für die Erforschung der Mechanismen von neuromuskulären Erkran- 
kungen als auch für die Entwicklung und das Screening von potenziellen therapeutischen Ansätzen (Abb. 3). Durch Verwendung von induzierten pluripotenten Stammzellen (iPSZellen) von Patienten mit neuromuskulären Erkrankungen, ist es möglich NMOs zu generieren, in denen alle Zelltypen patientenspezifisch sind und so Modelle zum Zweck der personalisierten Medizin mit Einzelzell-RNASequenzierung zu kombinieren. Die große Stärke des komplexen Organoidmodells ist die Möglichkeit, die Pathogenese in einem humanen, funktional manipulierbaren Modell zu untersuchen. Dies wird es erlauben, das primäre Angriffsziel der Krankheiten zu identifizieren und die Entwicklung von zielgerichteten Therapien zu erleichtern. Darüber hinaus bietet die Langzeitkultivierung Einblicke in frühe sowie auch späte Phänotypen in der Krankheitsentwicklung und macht damit auch die Modellierung von neuromuskulären Erkrankungen mit späten Krankheitsausbrüchen möglich.

\section{Ausblick}

Zukünftig kann die Komplexität der NMOs durch die Weiterentwicklung der Kulturbedingungen zusätzlich erhöht werden, um NMOs mit anterior-posterior-Identitäten zu entwickeln und somit alle Segmente des Rückenmarks und der assoziierten Muskulatur nachbilden zu können. Das erlaubt uns die jeweiligen Anfälligkeiten der Motorneuronen spezifischer Rückenmarkssegmente gegenüber neuromuskulären Krankheiten zu untersuchen. Bei der spinalen Muskelatrophie beispielsweise sind ausschließlich die Motorneuronen unterer Segmente betroffen, während die oberen intakt bleiben. Im Gegensatz dazu sind in den meisten ALSFällen Motorneuronen des oberen als auch unteren Segments betroffen, obwohl überraschenderweise die Funktion der Harnblase und des Darms sowie die Bewegung der Augen davon verschont bleibt [13]. Die Mechanismen, die der Besonderheiten der neuromuskulären Erkrankungen zugrunde liegen, sind größtenteils ungeklärt. Dies liegt hauptsächlich an der mangelnden Fähigkeit ausreichende Mengen an spezifischen Motorneuron- und Muskeltypen zu gewinnen, um mechanistische Studien durchführen zu können. Daher ist die Erzeugung beider Zelltypen mit präziser kompartimentärer Identität aus humanen iPS-Zellen notwendig, um die Mechanismen der neuromuskulären Krankheiten zweckmäßig zu erforschen. Um das Heranreifen der NMOs zu begünstigen,

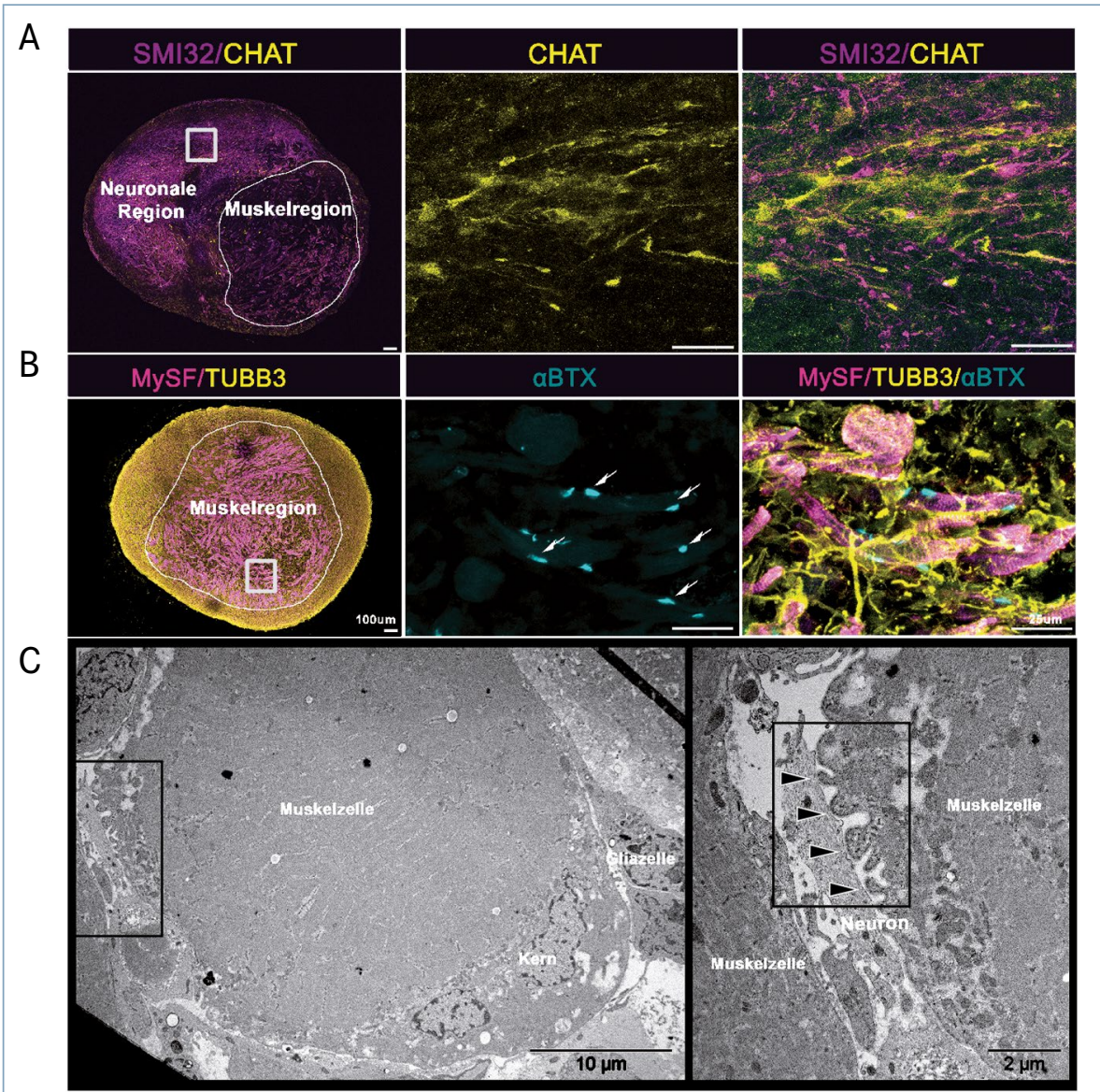

$\Delta$ Abb. 2: Schlüsselkomponenten der NMOs bleiben nach sechsmonatiger Kultivierung erhalten. A, Der reife Neurofilamentmarker SMI-32 wurde im neuralen Bereich des NMO detektiert. ChAT ${ }^{+}$ Motorneuronen (MN) sind in der Nähe der Skelettmuskelzellen organisiert. B, Axone der TUBB3 ${ }^{+}-$ Neuronen erstrecken sich durch Selbstorganisation in das Kompartiment der Skelettmuskelzellen, das durch myosin skeletal fast (MySF) gekennzeichnet wurde. Ihr Kontakt resultiert in der Bildung von $\alpha$-Bungarotoxin ${ }^{+}(\alpha B$ BX)-AChR-Cluster, das charakteristisch für funktionale NMJs ist. C, Transmissionselektronenmikroskopische Aufnahmen von fünf Monate alten Organoiden zeigen die Bildung von NMJs. MN befinden sich in der Nähe der Muskelzelle und synaptische Spalten bilden sich an den Kontaktstellen. Schwarze Pfeile deuten auf Einstülpungen der Plasmamembran in der Muskelzelle an der Motorneuronen-Muskel-Kontaktstelle hin.

ist der Input des Motorkortex notwendig. Daher könnte es möglich sein, durch Zusammensetzen von NMOs mit unterschiedlichen anterior-posterior-Identitäten und zerebralen Organoiden die komplexen Netzwerke zwischen den verschiedenen Regionen des zentralen Nervensystems in einem in vivo-ähnlichen Kontext zu erforschen.

Zusammenfassend präsentieren unsere Daten NMOs als ein spannendes neues Modell, um ein besseres Verständnis der Mechanismen von neuromuskulären Erkrankungen zu erlangen. Um die Entwicklung von zielgerichteten Therapien zu verbessern, könnten NMOs zur Etablierung einer Plattform für Wirkstoffscreenings verwendet werden, wo Kandidaten für therapeutische Anwendungen validiert und die Langzeiteffi- zienz sowie Sicherheit von neuen, in der präklinischen Testphase befindlichen Behandlungen geprüft werden. Das erlaubt uns ebenfalls die Identifikation früher Zeitpunkte für eine klinische Intervention vor Einsetzen von Symptomen und des kritischen Zeitfensters, in welchem diese Wirkstoffe die Motorneurondegeneration verhindern. Abschließend könnten NMOs der Wendepunkt zur Untersuchung von neuromuskulären Krankheiten sein. Ihre Anwendung in verschiedenen Krankheitskontexten könnten das neuromuskuläre Stammzellforschungsfeld enorm bereichern und bietet eine einfach zu bedienende, robuste Plattform für Wirkstoffscreenings zur Optimierung personalisierter Therapieoptionen für Patienten. 


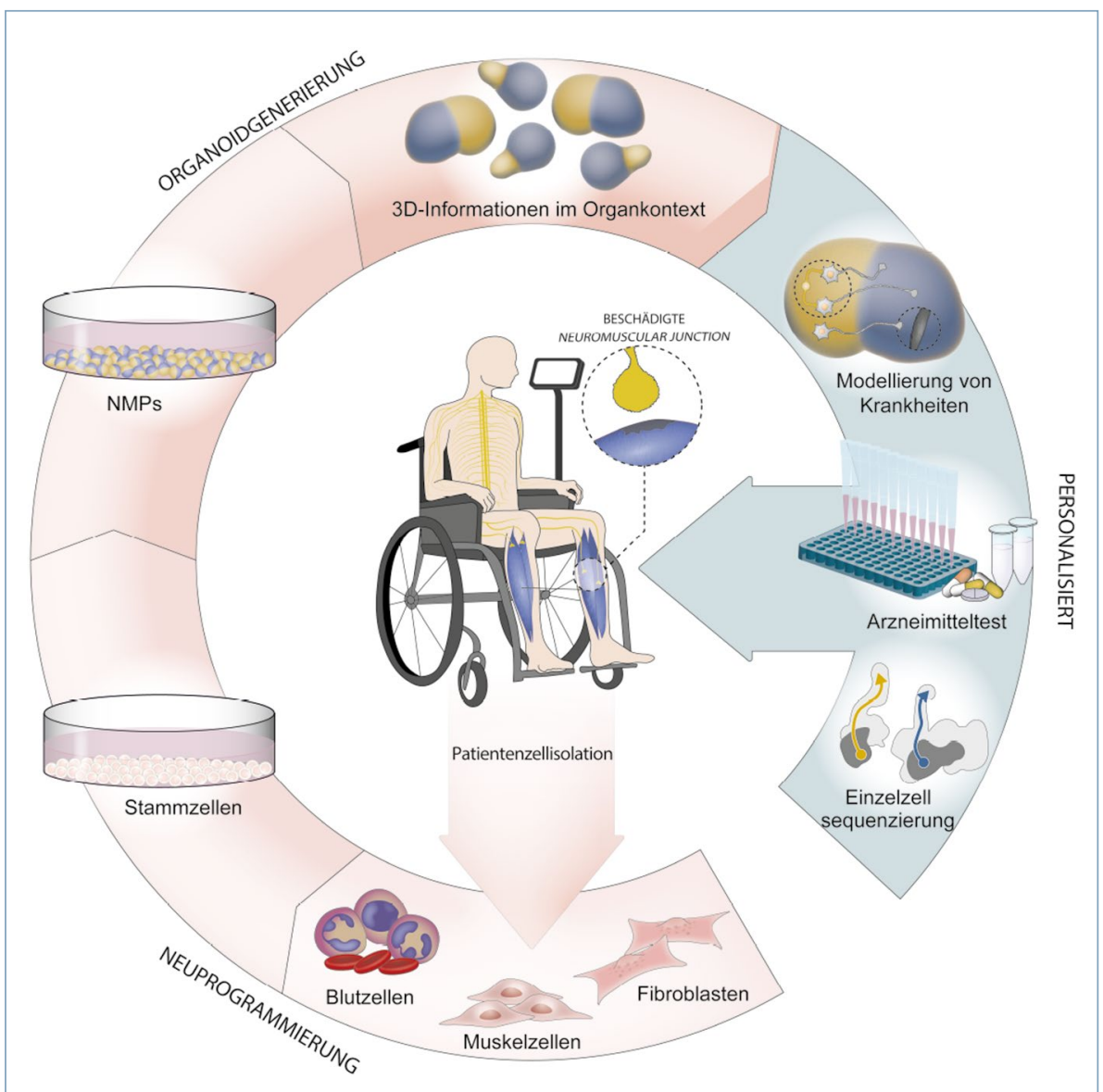

$\Delta$ Abb. 3: Zukünftige Anwendungsmöglichkeiten von NMOs in der personalisierten Medizin und in der Wirkstoffentwicklung. Aus Patienten mit neuromuskulären Erkrankungen isolierte Zellen können in induzierte pluripotente Stammzellen (iPS-Zellen) reprogrammiert werden. Die Generierung von NMOs aus patientenspezifischen iPS-Zellen erlaubt die Erforschung von Krankheitsmechanismen in einem humanen 3D-NMJ-Modell. Des Weiteren könnten mechanistische Hinweise aus Untersuchungen des transkriptomischen Profils patientenspezifischer NMOs auf Einzelzellebene erlangt werden. Durch die Verwendung von NMOs als Plattform für zielgerichtetes Wirkstoffscreening können Wirkstoffe in einem relevanten in vitro-Modell getestet werden, womit die Effizienz von verfügbaren therapeutischen Optionen für Patienten mit neuromuskulären Erkrankungen erhöht werden kann.

\section{Danksagung}

Wir bedanken uns bei der Elektronenmikroskopie-Plattform des MDC (Bettina Purfürst), insbesondere Séverine Kunz, für die elektro- nenmikroskopische Analyse. Außerdem danken wir Matthias Schmitt für die Revision des Manuskripts.

\section{Literatur}

[1] Lancaster MA, Renner M, Martin CA et al. (2013) Cerebral organoids model human brain development and microcephaly. Nature 501: 373-379

[2] Pasca AM, Sloan SA, Clarke LE et al. (2015) Functional cortical neurons and astrocytes from human pluripotent stem cells in 3D culture. Nat Methods 12: 671-678

[3] Faustino Martins JM, Fischer C, Urzi A et al. (2020) Selforganizing 3D human trunk neuromuscular organoids. Cell Stem Cell 26: 172-186

[4] Tzouanacou E, Wegener A, Wymeersch FJ et al. (2009) Redefining the progression of lineage segregations during mammalian embryogenesis by clonal analysis. Dev Cell 17: $365-376$

[5] Henrique D, Abranches E, Verrier L et al. (2015)

Neuromesodermal progenitors and the making of the spinal cord. Development 142: 2864-2875

[6] Kölliker A (1884) Die embryonalen Keimblätter und die Gewebe. Z Wiss Zool 40: 179-213

[7] Nieuwkoop PD (1954) Neural activation and transformation in explants of competent ectoerm under the influence of fragments of anterior notochord in urodeles. J Embryol Exp Morphol 2: 175-193

[8] Kimelman D (2016) Tales of tails (and trunks): forming the posterior body in vertebrate embryos. Curr Top Dev Biol 116: 517-536

[9] Gouti M, Tsakiridis A, Wymeersch FJ et al. (2014) In vitro generation of neuromesodermal progenitors reveals distinct roles for wnt signalling in the specification of spinal cord and paraxial mesoderm identity. PLoS Biol 12: e1001937

[10] Gouti M, Delile J, Stamataki D et al. (2017) A gene regulatory network balances neural and mesoderm specification during vertebrate trunk development. Dev Cell 41: 243-261 [11] Toyka KV, Drachman DB, Griffin DE et al. (1977) Myasthenia gravis. Study of humoral immune mechanisms by passive transfer to mice. N Engl J Med 296: 125-131 [12] Sanes JR, Lichtman JW (1999) Development of the vertebrate neuromuscular junction. Annu Rev Neurosci 22: 389442

[13] Kiernan MC, Vucic S, Cheah BC et al. (2011) Amyotrophic lateral sclerosis. Lancet 377: 942-955

Funding note: Open Access funding enabled and organized by Projekt DEAL. Open Access: Dieser Artikel wird unter der Creative Commons Namensnennu 4.0 International Lizenz veröffentlicht, welche die Nutzung, Vervielfältigung, Bearbeitung, Verbreitung und Wiedergabe in jeglichem Medium and ordnungsgemäß nennen, einen Link zur Creative Commons Lizenz beifügen und angeben, ob Änderungen vorgenten enthaltenen Bilder und sonstiges Drittmaterial unterliegen ebenfalls der genannten Creative Commons Lizenz, sofern sich aus der Abbildungslegende nichts anderes ergibt. Sofern das betreffende Material nicht unter der genannten Creative Commons Lizenz steht und die betreffende Handlung nich nach gesetzlichen Vorschriften erlaubt ist, ist für die oben aufgeführten Weiterverwendungen des Materials die Elinwiligung des jeweiligen Rechteinhabers einzuholen. Weitere Details zur Lizenz entnehmen Sie bitte der
Lizenzinformation auf htp: //creativecommons.org/licenses/by/4.0/deed.de.

\section{Korrespondenzadresse:}

\section{Dr. Mina Gouti}

Max Delbrück Centrum für Molekulare Medizin in der Helmholtz-Gemeinschaft

Robert-Rössle-Straße 10

D-13125 Berlin

mina.gouti@mdc-berlin.de www.mdc-berlin.de/de/gouti

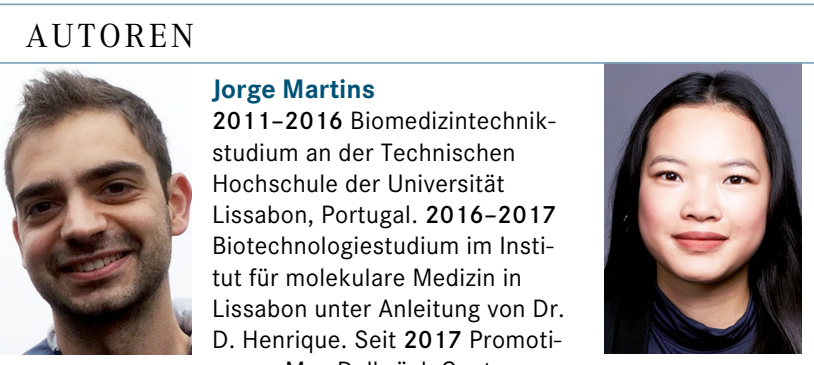

on am Max Delbrück Centrum Berlin unter Anleitung von Dr. M. Gouti.

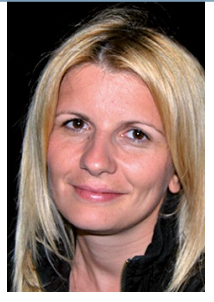

Mina Gouti

2012-2016 Wissenschaftlerin am Francis Crick Institute in London, UK. Seit 2016 Gruppenleiterin am Max Delbrück Centrum für Molekulare Medizin in der Helmholtz Gemeinschaft, Berlin. 2020 ERC Consolidator Grant zur Generierung positionsspezifische Organoide (GPSOrganoide) und Identifikation neuartiger Behandlungen für neuromuskuläre Erkrankungen. Auszeichnung als EMBO Young Investigator. 